\title{
A Selective Advantage to Immigrant Genes in a Daphnia Metapopulation
}

\author{
Dieter Ebert, ${ }^{1,2,3 *} \dagger$ Christoph Haag, ${ }^{1,2,3 *}$ Mark Kirkpatrick, ${ }^{4}$ \\ Myriam Riek, ${ }^{1,2}$ Jürgen W. Hottinger, ${ }^{1,2,3}$ V. Ilmari Pajunen ${ }^{2,5}$
}

Immigrants to habitats occupied by conspecific organisms are usually expected to be competitively inferior, because residents may be locally adapted. If residents are inbred, however, mating between immigrants and residents results in offspring that may enjoy a fitness advantage from hybrid vigor. We demonstrate this effect experimentally in a natural Daphnia metapopulation in which genetic bottlenecks and local inbreeding are common. We estimate that in this metapopulation, hybrid vigor amplifies the rate of gene flow several times more than would be predicted from the nominal migration rate. This can affect the persistence of local populations and the entire metapopulation.

Gene flow between populations can be both a creative and a constraining force in evolution (1-3). The introduction of new genetic material into a population increases local genetic diversity and helps the spread of favorable alleles across metapopulations. On the other hand, it reduces genetic variation between populations

'Zoologisches Institut, Universität Basel, Rheinsprung 9, 4051 Basel, Switzerland. ${ }^{2}$ Tvärminne Zoological Station, SF-10900 Hanko, Finland. ${ }^{3}$ Université de Fribourg, Département de Biologie, Unité d'Écologie et Évolution, Chemin du Musée 10, 1700 Fribourg, Switzerland. ${ }^{4}$ Section of Integrative Biology, University of Texas, Austin, TX 78712, USA. ${ }^{5}$ Division of Population Biology, Department of Ecology and Systematics, University of Helsinki, Post Office Box 17 (Arkadiankatu 7), SF-00014 University of Helsinki, Finland.

*These authors contributed equally to this work. $\dagger$ To whom correspondence should be addressed. Email: dieter.ebert@unifr.ch and hinders local adaptation. In inbred populations, the consequences of migration may be particularly important: If the hybrid offspring of immigrants and residents are competitively superior, their hybrid vigor will amplify the gene flow caused by migration (4-6). Furthermore, the demographic consequences of increased vigor could prevent the decline and even the extinction of populations (7-12). The magnitude of hybrid vigor is, however, controversial. Highly inbred populations may have low genetic loads because inbreeding exposes recessive deleterious alleles to purging by natural selection (13-17).

In subdivided populations with local extinctions and colonizations, genetic bottlenecks can be frequent, leading to increased homozygosity $(15,16,18)$. If homozygosity results in a fitness reduction (inbreeding de- 
pression), then small amounts of immigration can have disproportional effects on the vigor and persistence of local populations. This will in turn influence the extinction and colonization dynamics of the whole metapopulation (4-6).

To test whether hybrid vigor has an effect on the genetic structure of local populations, we studied the consequences of immigration in small populations of the water flea Daphnia magna. This planktonic crustacean occurs in various bodies of water, ranging from large ponds, with genetically diverse populations, to small intermittent pools, characterized by low genetic diversity. In our metapopulation, D. magna inhabits small pools $\left(0.5\right.$ to $20 \mathrm{~m}^{2}$ and 0.1 to $0.5 \mathrm{~m}$ deep) on the rocky banks of islands along the Swedish and Finnish Baltic Sea coast (19-22). The number of these rock pool populations is thought to be larger than $10^{5}$, with one to a few hundred pools per island. In our study area around Tvärminne in southern Finland, local extinction rates are about $20 \%$ per year, estimated over an 18 year study including 507 pools $(21,23)$. About $5 \%$ of the empty pools are colonized per year, and the proportion of occupied pools has remained around 20\% since 1983 . The life cycle of $D$. magna begins with the hatching of females from resting eggs in spring, followed by asexual reproduction for up to 12 generations. At the end of the season, sexual reproduction produces resting eggs that survive the winter and also serve as dispersal stages.

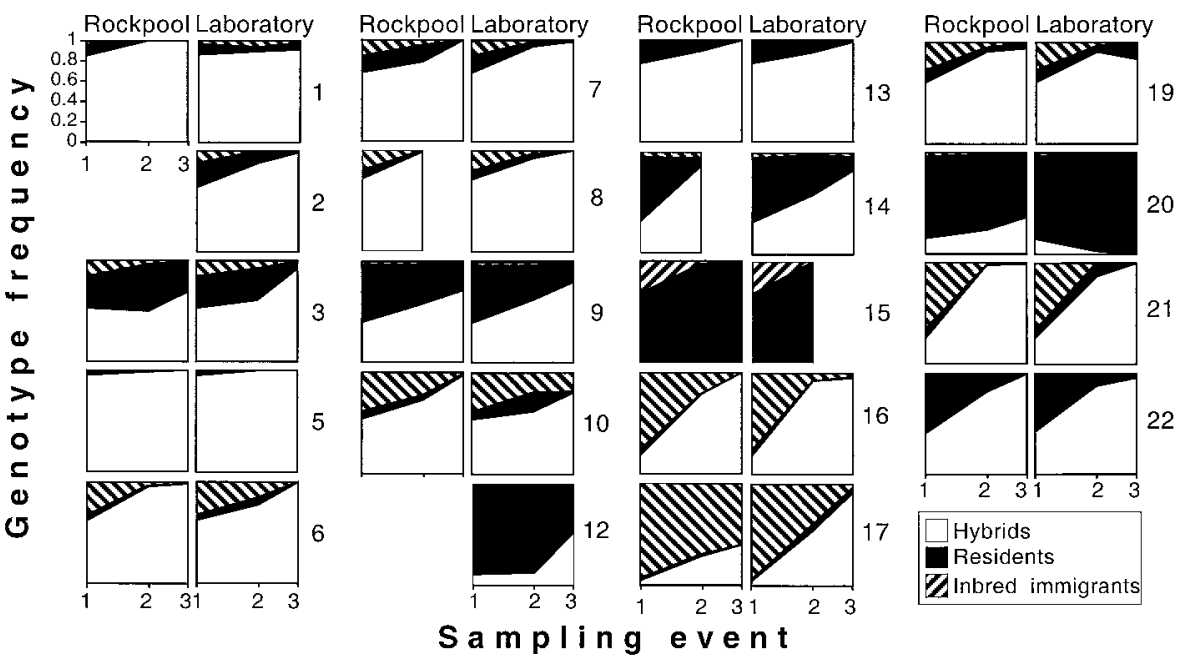

Fig. 1. Frequency changes of hybrids, residents, and inbred immigrants during asexual competition. The left plot of each pair of area plots shows frequency changes in the natural rock pools, whereas the right plots show changes in the laboratory. The population numbers are given on the right. All plots have the same $y$ axis (genotype frequencies ranging from 0 to 1 ) and $x$ axis (sampling events at 0,60 , and 100 days). The first sampling date was between 18 May and 5 June 2000 (depending on the emergence of Daphnia from resting eggs), the second between 18 and 21 July 2000, and the third on 28 August 2000. Empty places for two of the rock pool area plots and area plots including only the first and the second sample indicate extinction of these populations after the first or the second sample, respectively. Only 19 of the 22 populations are shown, because populations 4 and 11 went extinct during the winter and in population 18 only residents were found (sample size $>300$ ). No hybrids were detected in population 15 and no inbred immigrants in populations 12,13 , and 22 . In all other populations, we found all three offspring types. pools. Comparable disturbances occur naturally in this metapopulation, where storms on the Baltic Sea frequently wash out parts of or even entire pools $(19,21)$. We also collected 22 clones from different pools within the study area and propagated them in the laboratory. These we designated as our experimental immigrant clones.

Two weeks later, after rain had refilled the pools, we brought 200 individuals of the populations back into their original pools and added 200 individuals of one immigrant clone into each pool (26). In each experimental pool, the immigrant clone differed from the local clones at at least one allozyme marker locus. To avoid bias due to potential fitness effects of the genetic markers, we used multiple alleles at four loci. The genetic markers allowed us to distinguish between hybrids, offspring of local residents, and selfed offspring of the immigrant clone after the populations had undergone one round of sexual recombination, i.e., in spring of the following year.

Rock pools were left undisturbed until we took the first samples in May 2000. From these samples, we genotyped 66 to 122 animals and also founded laboratory populations using 200 to 300 females each. This was done to duplicate the natural rock pool experiment under controlled laboratory conditions so that we could decouple the effect of inbreeding from uncontrolled environmental effects, such as the local pool environment, predators, and further immigrants from the same or other Daphnia species (27). Second and third samples were taken from all populations about 60 and 100 days after the first samples had been collected.

Our hypothesis was that the outbred offspring would increase in frequency as the result of hybrid vigor. Figure 1 shows that this was the case in all rock pool populations where we recovered hybrids in May 2000 (all but pool 15). It was also the case in 17 of 18 laboratory populations (Fig. 1) (28). The changes in genotype frequencies in the field and the laboratory were highly correlated with each other $(r=0.71$ to 0.93 for the three offspring types, $P<0.002$, $n=16$ ), and the increase of outbred genotypes did not differ significantly among them (paired $t$ test: $t=0.52, P=0.61, n=$ 16). Hybrids also increased significantly in the time period between the second and the third sample $(P<0.01$ in the field and the laboratory) at a time when genotypes with very low fitness (e.g., due to castrating homozygous recessive mutations) had already been purged. Therefore, the observed hybrid vigor is at least partially due to deleterious effects of alleles with weak to intermediate effect $(6,29)$. As selection against such alleles is weak, they can accumulate to high frequencies. High genetic 
loads have been reported for Daphnia (3032).

We believe that hybrid vigor best explains our results, as a number of alternative hypotheses can be excluded. First, because we used a different immigrant clone for each population, it is unlikely that all of them carried superior alleles (33). Second, although rare genotypes could have had an advantage by, for example, exploiting unoccupied niches, we rejected this hypothesis because hybrids increased in frequency irrespective of their initial frequencies in May 2000 (Fig. 1). Third, although residents may suffer from locally adapted predators or parasites $(34,35)$, predators were excluded from the laboratory, and the increase of hybrids did not differ significantly between populations infected with a parasite or not (36). Fourth, mating success cannot explain our results (3739) as hybrid success was measured during the asexual phase of the Daphnia life cycle.

One key effect of hybrid vigor is an increase in effective gene flow (4). We estimate that 2 years after a natural immigration event (where immigrants represent a much smaller fraction of the population than in our experiments), the effective rate of gene flow is about 35 times larger than would be predicted by the number of immigrants alone, and it will even increase further in the following years (40). The amount by which gene flow is amplified depends on the causes and magnitude of hybrid vigor. It may be much smaller in genetically more diverse Daphnia populations but may be high in other systems characterized by frequent extinctions and recolonizations.

Amplified gene flow caused by hybrid vigor may account for lower observed levels of population differentiation than predicted by models based on neutral effects $(4,6$, 41-43) and may influence the evolution of dispersal (44). An important effect of hybrid vigor is the "genetic rescue" of populations from extinction, because it may influence extinction and colonization dynamics of the whole metapopulation (5). Although we did not show a link between population persistence and inbreeding depression, such a relation has been shown before $(9,11,12)$ and is likely to apply to Daphnia metapopulations as well. In this light, gene flow is an essential component for the persistence of metapopulations. Thus, our study gives clear empirical support for the need to maintain gene flow in the management and conservation of subdivided populations.

\section{Reference and Notes}

1. P. R. Ehrlich, P. H. Raven, Science 165, 1228 (1969).

2. E. Mayr, Animal Species and Evolution (Harvard Univ. Press, Cambridge, MA, 1963).

3. M. Slatkin, Science 236, 787 (1987).

4. P. K. Ingvarsson, M. C. Whitlock, Proc. R. Soc. London Ser. B 267, 1321 (2000).

5. C. M. Richards, Am. Nat. 155, 383 (2000).
6. M. C. Whitlock, P. K. Ingvarsson, T. Hatfield, Heredity 84, 452 (2000).

7. R. Lande, Evolution 48, 1460 (1994).

8. M. Lynch, J. Cornery, R. Bürger, Am. Nat. 146, 489 (1995).

9. I. M. Saccheri et al., Nature 392, 491 (1998).

10. R. Frankham, Conserv. Biol. 12, 665 (1998).

11. R. L. Westemeier et al., Science 282, 1695 (1998).

12. T. Madsen, R. Shine, M. Olsson, H. Wittzell, Nature 402, 34 (1999).

13. R. Lande, D. W. Schemske, Evolution 39, 24 (1985).

14. S. C. H. Barrett, D. Charlesworth, Nature 352, 522 (1991).

15. L. F. Keller, J. N. Arcese, M. Smith, W. M. Hochachka, S. C. Stearns, Nature 372, 356 (1994).

16. D. Charlesworth, B. Charlesworth, Annu. Rev. Ecol. Syst. 18, 237 (1987).

17. E. H. Bryant, L. M. Meffert, L. M. McCommas, Am. Nat. 136, 542 (1990).

18. C. R. Darwin, The Effects of Cross and Self Fertilization in the Vegetable Kingdom (John Murray, London, 1876).

19. E. Ranta, Arch. Hydrobiol. 87, 205 (1979).

20. I. Hanski, E. Ranta, J. Anim. Ecol. 52, 263 (1983).

21. V. I. Pajunen, Ann. Zool. Fennici 23, 131 (1986).

22. S. Ås, J. Bengtsson, T. Ebenhard, in Ecological Principles of Nature Conservation, L. Hansson, Ed. (Elsevier, London, 1992), pp. 201-251.

23. V. I. Pajunen, I. Pajunen, unpublished data

24. In 1998, we genotyped 22 to 44 D. magna from all 96 pools (out of 507) occupied in the study region at five polymorphic loci using allozyme electrophoresis (45). Twenty-nine independent (i.e., pools not connected to other pools) new colonizations were recorded in this summer, none of which was polymorphic at more than two loci. In 26 of these populations, only one genotype was found. Of these, five were fixed at a heterozygote locus, suggesting that they may have been colonized by only one clone each. One population was composed of two heterozygote clones (genotypes $\mathrm{AA} / \mathrm{Bb}$ and $\mathrm{Aa} / \mathrm{BB}$ ) with strong linkage disequilibrium $(P<0.01)$, suggesting that it may have been colonized by two polymorphic clones. The finding of five fixed heterozygotes among 29 newly colonized populations is in contrast to the finding of only one fixed heterozygotes among the 67 older populations (colonized in 1997 or earlier) that underwent at least one season of sexual reproduction $(P=$ 0.009 , Fisher exact test). At least three of the 29 new populations resulted from interisland migration, because no other $D$. magna populations were present on these islands during previous years.

25. With selfing, the inbreeding coefficient $F$ of the entire population will be at least 0.50 in the following year. As resting eggs usually come in pairs, founding events may include two clones that are related to each other as full or half sibs. Then $F$ in the following year would be at least 0.25 or 0.125 , respectively.

26. Under natural conditions, the number of immigrants is likely to be very small relative to the number of residents. To minimize stochastic events influencing the fate of the immigrants during our experiment, we introduced the local residents and the immigrants in equal numbers.

27. Laboratory populations were kept in white buckets filled with 8 liters of artificial medium. Populations were kept at $18^{\circ} \mathrm{C}$ and $18: 6$ hours light:dark cycle and were fed daily with $1.5 \times 10^{7}$ cells of Scenedesmus gracilis. Five liters of the medium was exchanged after 6 and 11 weeks. Population size was 250 to 400 . Predators and competitors were excluded from these populations.

28. The genotype frequency differences between the last successful sample (third sample, unless extinction occurred after the second sample) and the first sample were tested with a Wilcoxon sign rank test (paired test). While the hybrids increased significantly (field: $Z=68, P<0.0001, n=16$; laboratory: $Z=$ 82.5, $P<0.0001, n=18$ ), the residents (field: $Z=$ $-60, P<0.0001, n=16$; laboratory: $Z=-65.5, P=$ $0.0028, n=18$ ) and the selfed immigrant offspring decreased significantly (field: $Z=-44.5, P=0.0005$, $n=15$; laboratory: $Z=-60, P<0.0001, n=16$ ). Tests excluded population 15 , because no hybrids were found.
29. B. Charlesworth, D. Charlesworth, M. T. Morgan, Nature 347, 380 (1990).

30. D. J. Innes, J. Hered. 80, 6 (1989).

31. L. De Meester, Oecologia 96, 80 (1993)

32. M. Lynch, H. W. Deng, Am. Nat. 144, 242 (1994).

33. The genetic markers used to trace the genotypes during asexual competition had no influence on the advantage of the hybrids relative to the residents [analysis of variance; field: $F(3,7)=0.27, P=0.85$; laboratory: $F(3,11)=1.49, P=0.27$ ]

34. D. Ebert, Science 265, 1084 (1994).

35. C. C. Wilson, P. D. N. Hebert, Ecology 73, 1462 (1992).

36. Fourteen populations in the field and 12 populations in the laboratory harbored at least one out of three naturally occurring parasites: two microsporidia and one bacterium. There was no significant difference in hybrid frequency increase between infected and uninfected populations [laboratory populations: $F(1,16)=0.97, P=0.34$; field: $F(1,14)=0.21, P=$ $0.65]$.

37. P. Sinnock, Am. Nat. 104, 469 (1970).

38. C. S. Jones et al., Proc. R. Soc. London Ser. B 260, 251 (1995).

39. L. De Meester, J. Vanoverbeke, Proc. R. Soc. London Ser. B 266, 2471 (1999)

40. The impact of hybrid vigor on gene flow can be quantified by the "effective migration rate" $m^{*}(t)$ defined as the migration rate that would have the same impact on the genetic composition of the population as an equivalent number of copies of a migrant gene $t$ years after it arrives if hybrid vigor was absent. The following argument applies to selected as well as neutral genes. In the Daphnia metapopulation, migrants arrive as resting eggs before the growing season of year 1 or as asexual females during it. They form clones during year 1 that mate with residents to produce $F$ hybrid resting eggs. At the end of year $1, m^{*}(1)=m$ because hybrid vigor is not yet expressed. If the number of immigrants is small relative to residents and Daphnia mate randomly or disassortatively, then virtually all immigrants mate with residents. Thus, $m^{*}(2)=w_{F 1} m$, where $w_{F 1}$ is the mean fitness of the hybrids relative to residents. From our data during the 100-day study period (representing about one sexual generation), we estimate $m^{*}(2)=33.7 m$ using the laboratory data and $36.0 \mathrm{~m}$ using the field data. The fitness of hybrids was estimated as

$$
W_{F 1}=\left[\left(1-p_{1}\right) p_{3}\right] /\left[p_{1}\left(1-p_{3}\right)\right]
$$

where $p_{1}$ and $p_{3}$ are the frequencies of the hybrids at the beginning and end of the experiment (about one sexual generation). As replicates in which $p_{3}=1$ (hybrids went to fixation) were not included, our estimates of $w_{F 1}$ are underestimates. In subsequent years, $m^{*}$ can be determined for genes that are unlinked to other loci under selection: $m^{*}(t)=$ $W_{F 1} W_{B(1)} W_{B(2)} \ldots W_{B(t-2)} m$, where $w_{B(1)}$ is the mean fitness of individuals resulting from the first backcross between the $F_{1}$ and residents and $W_{B(i)}$ is the mean fitness of offspring from backcross $i-1$ and residents. This expression for $m^{*}$ is an approximation that holds so long as the frequency of matings between backcross individuals is negligible in all years. The value of $m^{*}(t)$ will grow in successive years if the backcrosses have higher fitness than the residents, reaching an asymptote as the population reaches linkage equilibrium. We do not have the fitnesses of the backcrosses, and so we calculate $m^{*}$ using a simple model that assumes that fitness differences are caused by deleterious mutations of equal effect at unlinked loci. Assuming that the mutations have multiplicative effects, then the fitness of a genotype can be described by the numbers of homo- and heterozygous mutants that they carry. We write the relative fitness effects of a single locus as $1,(1-h s)$, and $(1-s)$ for genotypes with 0,1 , and 2 copies of a mutation. Assume that migrants and residents each consist of a single clone that is entirely homozygous, with the migrants and residents fixed for alternative alleles at $i_{m}$ and $i_{r}$ loci, respectively. Calculations then show that 


$$
\begin{gathered}
W_{F 1}=\frac{(1-h s)^{\left(i_{m}+i_{f}\right)}}{(1-s)^{i_{f}}}, \\
W_{B(1)}=\left(1-\frac{1}{2} h s\right)^{i_{m}}\left[\frac{2-s-h s}{2(1-s)}\right]^{i_{F}}
\end{gathered}
$$

and that the fitnesses of later backcrosses converge geometrically on 1 at a rate of $1 / 2$ per sexual generation (year). Using parameter values suggested for Daphnia (46) $(s=0.14$ and $h=0.3)$ and setting $i_{m}=i_{r}=55$ to give an $F_{1}$ fitness similar to what we observed, we obtain $m^{*}(2)=35.7 m$, $m^{*}(3)=234 m$, and $m^{*}(\infty)=6703 m$. Lower values of $m^{*}$, but still substantially greater than 1 , can be obtained when $i_{m}$ and $i_{r}$ have more modest values. Thus, hybrid vigor can make gene flow many times stronger than would be predicted from the nominal migration rate under these conditions.

41. P. Pamilo, S. Pálsson, O. Savolainen, Hereditas 130, 257 (1999).

42. B. Charlesworth, M. Nordborg, D. Charlesworth, Genet. Res. Camb. 70, 155 (1997).

43. A. G. Young, T. Boyle, T. Brown, Trends Ecol. Evol. 11, 413 (1996).

44. I. Olivieri, P. H. Gouyon, in Metapopulation Biology,
I. A. Hanski, M. E. Gilpin, Eds. (Academic Press, San Diego, CA, 1997), pp. 293-324.

45. P. D. N. Hebert, M. J. Beaton, Methodologies for Allozyme Analysis Using Cellulose Acetate Electrophoresis (Helena Laboratories, Beaumont, TX, ed. 2, 1993).

46. H. W. Deng, M. Lynch, Genetics 147, 147 (1997).

47. We thank T. Kawecki, M. Ackermann, D. Meyer, and S. Zweizig for critical comments to earlier version of the manuscript. This work was supported by the Swiss Science Foundation and NSF grant DEB-9973221 